\title{
LXXVI. Experimental researches in electricity.-Twentieth series
}

\section{Michael Faraday Esq. D.C.L. F.R.S.}

To cite this article: Michael Faraday Esq. D.C.L. F.R.S. (1846) LXXVI. Experimental researches in electricity.-Twentieth series, Philosophical Magazine Series 3, 28:189, 455-468, DOI: $10.1080 / 14786444608645459$

To link to this article: http://dx.doi.org/10.1080/14786444608645459

册 Published online: 30 Apr 2009.

Submit your article to this journal $\lceil\pi$

Џ Article views: 7

Q View related articles $\square$

Citing articles: 1 View citing articles 5 
animal frame, is capable of being applied in producing a useful mechanical effect, - the remaining three-quarters being required in order to keep up the animal heat, \&c.

Prof. Magnus of Berlin, has endeavoured to prove that the oxygen which an animal inspires does not combine chemically with the blood, but is merely absorbed by it*. The blood thus charged with oxygen arrives in the capillary vessels, where the oxygen effects a chemical combination with certain substances, converting them into carbonic acid and water. The carbonic acid, instead of oxygen, is then absorbed by the blood, and thus reaches the lungs to be removed by contact with the atmosphere. Adopting this view, it becomes exceedingly probable that the whole of the vis viva due to the oxidation or combustion of the "certain substances" mentioned by Magnus is developed by the muscles. The muscles, by their motion, can communicate vis viva to external objects; and, by their friction within the body, can develope heat in various quantities according to circumstances, so as to maintain the animal at an uniform temperature. If these theoretic views be correct, they wonld lead to the interesting conclusion (which is the same as that announced by Matteucci from other considerations) that the animal frame, though destined to fulfill so many other ends, is, as an engine, more perfect in the oeconomy of vis viva than the best of human contrivances.

LXXVI. Experimental Researches in Electricity.-Twentieth Series. By Mrchael Faraday, Esq., D.C.L., F.R.S., Fullerian Prof., \&c. \&.c.

[Concluded from p. 406.]

$T$ iv. Action of magnets on metals generally.

2287. r THE metals, as a class, stand amongst bodies having 1 a high and distinct interest in relation both to magnetic and electric forces, and might at first well be expected to present some peculiar phænomena, in relation to the striking property found to be possessed in common by so large a number of substances, so varied in their general characters. As yet no distinction associated with conduction or non-conduction, transparent or opake, solid or liquid, crystalline or amorphous, whole or broken, has presented itself; whether the metals, distinct as they are as a class, would fall into the great generalization, or whether at last a separation would occur, was to me a point of the highest interest.

2288. That the netals, iron, nickel and cobalt, would stand in a distinct class, appeared almost undoubted; and it will be,

* [See Phil. Mag. S. 3, vol. xxvii. p. 561.] 
I think, for the advantage of the inquiry, that I should consider them in a section apart by themselves. Further, if any other metals appeared to be magnetic, as these are, it would be right and expedient to include them in the same class.

2289. My first point, therefore, was to examine the metals for any indication of ordinary magnetism. Such an examination cannot be carried on by magnets anything short in power of those to be used in the further investigation; and in proof of this point I found many specimens of the metals, which appeared to be perfectly free from magnetism when in the presence of a magnetic needle, or a strong horse-shoe magnet (2157.), that yet gave abundant indications when suspended near to one or both poles of the magnets described (2246.).

2290 My test of magnetism was this. If a bar of the metal to be examined, about two inches long, was suspended (2249.) in the magnetic field, and being at first oblique to the axial line, was upon the supervention of the magnetic forces drawn into the axial position instead of being driven into the equatorial line, or remaining in some oblique direction, then I considered it magnetic. Or, if being near one magnetic pole, it was attracted by the pole, instead of being repelled, then I concluded it was magnetic. It is evident that the test is not strict, because, as before pointed out (2285.), a body may have a slight degree of magnetic force, and yet the power of the new property be so great as to neutralize or surpass it. In the first case, it might seem neither to have the one property nor the other; in the second case, it might appear free from magnetism, and possessing the special property in a small degree.

2291. I obtained the following metals, so that when examined as above, they did not appear to be magnetic; and in fact if magnetic, were so to an amount so small as not to destroy the results of the other force, or to stop the progress of the inquiry.

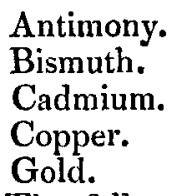

Lead. Mercury. Silver. Tin. Zinc.

2292. The following metals were, and are as yet to me, magnetic, and therefore companions of iron, nickel and cobalt :-

Platinum.

Palladium.

Titanium.

2293. Whether all these metals are magnetic, in consequence of the presence of a little iron, nickel, or cobalt in them, 
or whether any of them are really so of themselves, I do not undertake to decide at present; nor do I mean to say that the metals of the former list are free. I have been much struck by the apparent freedom from iron of almost all the specimens of zinc, copper, antimony and bismuth, which I have examined; and it appears to me very likely that some metals, as arsenic, \&c., may have much power in quelling and suppressing the magnetic properties of any portion of iron in them, whilst other metals, as silver or platinum, may have little or no power in this respect.

2294. Resuming the consideration of the influence excited by the magnetic force over those metals which are not magnetic after the manner of iron (2291.), I may state that there are two sets of effects produced which require to be carefully distinguished. One of these depends upon induced magnetoelectric currents, and shall be resumed hereafter (2309.). The other includes effects of the same nature as those produced with heavy glass and many other bodies (2276.).

2295. All the non-magnetic metals are subject to the magnetic power, and produce the same general effects as the large class of bodies already described. The force which they then manifest, they possess in different degrees. Antimony and bismuth show it well, and bismuth appears to be especially fitted for the purpose. It excels heavy glass, or borate of lead, and perhaps phosphorus; and a small bar or cylinder of it about two inches long, and from 0.25 to 0.5 of an inch in width, is as well fitted to show the various peculiar phænomena as anything I have yet submitted to examination.

2296. To speak accurately, the bismuth bar which I employed was two inches long, 0.33 of an inch wide, and 0.2 of an inch thick. When this bar was suspended in the magnetic field, between the two poles, and subject to the magnetic force, it pointed freely in the equatorial direction, as the heavy glass did (2253.), and if disturbed from that position returned freely to it. This latter point, though perfectly in accordance with the former phænomena, is in such striking contrast with the phænomena presented by copper and some other of the metals (2309.), as to require particular notice here.

2297. The comparative sensibility of bismuth causes several movements to take place under various circumstances, which being complicated in their nature, require careful analysis and explanation. The chief of these, with their causes, I will proceed to point out.

2298. If the cylinder electro-magnet (2246.) be placed ver. tically so as to present one pole upwards, that pole will exist in the upper end of an iron cylinder, having a flat horizontal Phil. Mag. S. 3. Vol. 28. No. 189. June 1846. 
face $2 \frac{1}{2}$ inches in diameter. A small indicating sphere (2266.) of bismuth hung over the centre of this face and close to it, does not move by the magnetism. If the ball be carried outwards, half way, for instance, between the centre and the edge, the magnetism makes it move inwards, or towards the axis (prolonged) of the iron cylinder. If carried still further outwards, it still moves inwards under the influence of the magnetism, and such continues to be the case until it is placed just over the edge of the terminal face of the core, where it has no motion at all (here, by another arrangement of the experiment, it is known to tend in what is at present an upward direction from the core). If carried a little further outwards, the magnetism then makes the bismuth ball tend to go outwards or be repelled, and such continues to be the direction of the force in any further position, or down the side of the end of the core.

2299. In fact, the circular edge formed by the intersection of the end of the core with its sides, is virtually the apex of the magnetic pole, to a body placed like the bismuth ball close to it, and it is because the lines of magnetic force issuing from it diverge as it were, and weaken rapidly in all directions from it, that the ball also tends to pass in all directions either inwards or upwards, or outwards from it, and thus produces the motions described. These same effects do not in fact all occur when the ball, being taken to a greater distance from the iron, is placed in magnetic curves, having generally a simpler direction. In order to remove the effect of the edge, an iron cone was placed on the top of the core, converting the flat end into a cone, and then the indicating ball was urged to move upwards, only when over the apex of the cone; and upward and outwards, as it was more or less on one side of it, being always repelled from the pole in that direction, which transferred it most rapidly from strong to weaker points of magnetic force.

2300. To return to the vertical flat pole: when a horizontal bar of bismuth was suspended concentrically and close to the pole, it could take up a position in any direction relative to the axis of the pole, having at the same time a tendency to move upwards or be repelled from it. If its point of suspension was a little excentric, the bar gradually turned, until it was parallel to a line joining its point of suspension with the prolonged axis of the pole, and the centre of gravity moved inwards. When its point of suspension was just outside the edge of the flat circular terminating face, and the bar formed a certain angle with a radial line joining the axis of the core and the point of suspension, then the movements of the bar were un- 
certain and wavering. If the angle with the radial line were less than that above, the bar would move into parallelism with the radius and go inwards: if the angle were greater, the bar would move until perpendicular to the radial line and go outwards. If the centre of the bar were still further out than in the last case, or down by the side of the core, the bar would always place itself perpendicular to the radius and go outwards. All these complications of motion are easily resolved into their simple elementary origin, if reference be had to the character of the circular angle bounding the end of the core; to the direction of the magnetic lines of force issuing from it and the other parts of the pole; to the position of the different parts of the bar in these lines; and the ruling principle that each particle tends to go by the nearest course from strong to reaker points of magnetic force.

2301. The bismuth points well, and is well repelled (2296.) when immersed in water, alcohol, æther, oil, mercury, \&c., and also when inclosed within vessels of earth, glass, copper, lead, \&c. (2272.), or when screens of 0.75 or 1 inch in thickness of bismuth, copper or lead intervene. Even when a bismuth cube (2266.) was placed in an iron vessel $2 \frac{1}{2}$ inches in diameter and 0.17 of an inch in thickness, it was well and freely repelled by the magnetic pole.

2302. Whether the bismuth be in one piece or in very fine powder, appears to make no difference in the character or in the degree of its magnetic property (2283.).

2303. I made many experiments with masses and bars of bismuth suspended, or otherwise circumstanced, to ascertain whether two pieces had any mutual action on each other, either of attraction or repulsion, whilst jointly under the influence of the magnetic forces, but I could not find any indication of such mutual action: they appeared to be perfectly indifferent one to another, each tending only to go from stronger to weaker points of magnetic power.

2304. Bismuth, in very fine powder, was sprinkled upon paper, laid over the horizontal circular termination of the vertical pole (2246.). If the paper were tapped, the magnet not being excited, nothing particular occurred; but if the magnetic power were on, then the powder retreated in both directions, inwards and outwards, from a circular line just over the edge of the core, leaving the circle clear, and at the same time showing the tendency of the particles of bismuth in all directions from that line (2299.).

2305. When the pole was terminated by a cone (2246.) and the magnet not in action, paper with bismuth powder sprinkled over it being drawn over the point of the cone, gave 
no particular result; but when the magnetism was on, such an operation cleared the powder from every point which came over the cone, so that a mark was traced or written out in clear lines running through the powder, and showing every place where the pole had passed.

2306. The bar of bismuth and a bar of antimony was found to set equatorially between the poles of the ordinary horseshoe magnet.

2307. The following list may serve to give an idea of the apparent order of some metals, as regards their power of producing these new effects, but I cannot be sure that they are perfectly free from the magnetic metals. In addition to that, there are certain other effects produced by the action of magnetism on metals (2309.) which greatly interfere with the results due to the present property.

\begin{tabular}{l|l} 
Bismuth. & Cadmium. \\
Antimony. & Mercury. \\
Zinc. & Silver. \\
Tin. & Copper.
\end{tabular}

2308. I have a vague impression that the repulsion of bismuth by a magnet has been observed and published several years ago. If so, it will appear that what must then have been considered as a peculiar and isolated effect, was the consequence of a general property, which is now shown to belong to all matter*.

2309. I now turn to the consideration of some peculiar phænomena which are presented by copper and several of the metals when they are subjected to the action of magnetic forces, and which so tend to mask effects of the kind already described, that if not known to the inquirer they would lead to much confusion and doubt. These I will first describe as to their appearances, and then proceed to consider their origin.

2310. If instead of a bar of bismuth (2296.) a bar of copper of the same size be suspended between the poles (224.7.), and

* M. de la Rive has this day referred me to the Bibliothèque Universelle for 1829 , tome xl. p. 82 , where it will be found that the experiment spoken of above is due to $M$. la Baillif of Paris. M. Ia Baillif showed sixteen years ago that both bismuth and antimony repelled the magnetic needle. It is astonishing that such an experiment has remained so long without further results. I rejoice that I am able to insert this reference before the present series of these researches goes to press. Those who read my papers will see here, as on many other occasions, the results of a memory which becomes continually weaker; I only hope that they will be excused, and that omissions and error's of that nature will be considered as involuntary.M. F. December 30, 1845. 
magnetic power be developed whilst the bar is in a position oblique to the axial and equatorial lines, the experimenter will perceive the bar to be affected, but this will not be manifest by any tendency of the bar to go to the equatorial line; on the contrary, it will advance towards the axial position as if it were magnetic. It will not however continue its course until in that position, but, unlike any effect produced by magnetism, will stop short, and making no vibration beyond or about a given point, will remain there coming at once to a dead rest: and this it will do even though the bar by the effect of torsion or momentum was previously moving with a force that would have caused it to make several gyrations. This effect is in striking contrast with that which occurs when antimony, bismuth, heavy glass, or other such bodies are employed, and it is equally removed from an ordinary magnetic effect.

2311. The position which the bar has taken up it retains with a considerable degree of tenacity, provided the magnetic force be continued. If pushed out of it, it does not return into it, but takes up its new position in the same manner, and holds it with the same stiffness; a push however, which would make the bar spin round several times if no magnetism were present, will now not move it through more than $20^{\circ}$ or $30^{\circ}$. This is not the case with bismuth or heavy glass; they vibrate freely in the magnetic field, and always return to the equatorial position.

2312. The position taken up by the bar may be any position. The bar is moved a little at the instant of superinducing the magnetism, but allowing and providing for that, it may be finally fixed in any position required. Even when swinging with considerable power by torsion or momentum, it may be caught and retained in any place the experimenter wishes.

2313. There are two positions in which the bar may be placed at the beginning of the experiment, from which the magnetism does not move it, the equatorial and the axial positions. When the bar is nearly midway between these, it is usually most strongly affected by the first action of the magnet, but the position of most effect varies with the form and dimensions of the magnetic poles and of the bar.

2314. If the centre of suspension of the bar be in the axial line, but near to one of the poles, these movements occur well, and are clear and distinct in their direction: if it be in the equatorial line, but on one side of the axial line, they are modified, but in a manner which will easily be understood hereafter.

2315. Having thus stated the effect of the supervention of 
the magnetic force, let us now remark what occurs at the moment of its cessation; for during its continuance there is no change. If, then, after the magnetism has been sustained for two or three seconds, the electric current be stopped, there is instantly a strong action on the bar, which has the appearance of a revulsion (for the bar returns upon the course which it took for a moment when the electric contact was made), but with such force, that whereas the advance might be perhaps $15^{\circ}$ or $20^{\circ}$, the revulsion will cause the bar occasionally to move through two or three revolutions.

2316. Heavy glass or bismuth presents no such phænomena as this.

2317. If, whilst the bar is revolving from revulsion the electric current at the magnet be renewed, the bar instantly stops with the former appearances and results (2310.), and then upon removing the magnetic force is affected again, and, of course, now in a contrary direction to the former revulsion.

2318. When the bar is caught by the magnetic force in the axial or equatorial position, there is no revulsion. When inclined to these positions there is; and the places most powerful in this respect appear to be those most favourable to the first brief advance (2313.). If the bar be in a position at which strong revulsion would occur, and whilst the magnetism is continued be moved by hand into the equatorial or axial position, then on taking off the magnetic force there is no revulsion.

2319. If the continuance of the electric current and consequently of the magnetism be for a moment only, the revulsion is very little, and the shorter the continuance of the magnetic force the less is the revulsion. If the magnetic force be continued for two or three seconds and then interrupted and in stantly renewed, the bar is loosened and caught again by the power before it sensibly changes its place; and now it may be observed that it does not advance on the renerval of the force as it would have done had it been acted on by a first contact in that place (2310.); $i$. $e$. if the bar be in a certain place inclined to the axial position, the first supervention of the magnetic power causes it to advance towards the axial position; but the bar being in the same place and the magnetic power suspended and instantly renewed, the second supervention of force does not move the bar as the first did.

2320. When the copper bar is immersed in water, alcohol, or even mercury, the same effects take place as in the air, but the movements are, of course, not to the same extent.

2321. When plates of copper or bismuth, an inch in thick- 
ness, intervene between the poles and the copper bar, the same results occur.

2322. If one magnetic pole only be employed the effects occur near it as well as before, provided that pole have a face large in proportion to the bar, as the end of the iron core (2246.): but if the pole be pointed by the use of the conical termination, or if the bar be opposite the edge of the end of the core, then they become greatly enfeebled or disappear altogether; and only the general fact of repulsion remains (2295.).

2323. The peculiar effects which have just been described are perhaps more strikingly shown if the bar of copper be suspended perpendicularly, and then hung opposite and near to the large face of a single magnetic pole, or the pole being placed vertically, as described (224.6. 2263.), anywhere near to its side. The bar, it will be remembered, is two inches in length by 0.33 of an inch in width, and 0.2 of an inch in thickness, and as it now will revolve on an axis parallel to its length, the two smaller dimensions are those which are free to move into new positions. In this case the establishment of the magnetic force causes the bar to turn a little in accordance with the effects before described, and the removal of the magnetic force causes a revulsion, which sends the bar spinning round on its axis several times. But at any moment the bar can again be caught and held in a position as before. The tendency on making contact at the battery is to place the longest moving dimension, i.e. the width of the bar, parallel to the line joining the centre of action of the magnet and the bar.

2324. The bar, as before (2311.), is extremely sluggish and as if immersed in a dense fluid, as respects rotation on its own axis; but this sluggrishness does not affect the bar as a whole, for any pendulum vibration it has continues unaffected. It is very curious to see the bar, jointly vibrating from its point of suspension (2249.) and rotating on its axis, when first affected by the magnetic force, for instantly the latter motion ceases, but the former goes on with undiminished power.

2325. The same effect of sluggishness occurs with a cube or a globe of copper as with the bar, but the phænomena of the first turn and the revulsion cease (2310. 2315.).

2326. The bars of bismuth and heavy glass present no appearance of this lsind. The peculiar phænomena produced by copper are as distinct from the actions of these substances as they are from ordinary magnetic actions.

2927. Endeavouring to explain the cause of these effects, it appear's to me that they depend upon the excellent con- 
ducting power of copper for electric currents, the gradual acquisition and loss of magnetic power by the iron core of the electro-magnet, and the production of those induced currents of magneto-electricity which I described in the First Series of these Experimental Researches (55. 309.).

2328. The obstruction to motion on its own axis, when the bar is subjected to the magnetic forces, belongs equally to the form of a sphere or a cube. It belongs to these bodies, however, only when their axes of rotation are perpendicular or oblique to the lines of magnetic force, and not when they are parallel to it; for the horizontal bar, or the vertical bar, or the cube or sphere, rotate with perfect facility when they are suspended above the vertical pole (2246.), the rotation and vibration being then equally free, and the same as the corresponding movements of bismuth or heavy glass. The obstruction is at a maximum when the axis of rotation is perpendicular to the lines of magnetic force, and when the bar or cube, \&c. is near to the magnet.

2329. Without going much into the particular circumstances, I may say that the effect is fully explained by the electric currents induced in the copper mass. By reference to the Second Series of these Researches $(160$.$) , it will be$ seen that when a globe, subject to the action of lines of magnetic force, is revolving on an axis perpendicular to these lines, an electric current runs round it in a plane parallel to the axis of rotation and to the magnetic lines, producing consequently a magnetic axis in the globe, at right angles to the magnetic curves of the inducing magnet. The magnetic poles of this axis therefore are in that direction which, in conjunction with the chief magnetic pole, tends to draw the globe back against the direction in which it is revolving. Thus, if a piece of copper be revolving before a north magnetic pole, so that the parts nearest the pole move towards the right-hand, then the right-hand side of that copper will have a south magnetic state, and the left-hand side a north magnetic state; and these states will tend to counteract the motion of the copper towards the right-hand: or if it revolve in the contrary direction, then the right-hand side will have a south magnetic state, and the left-hand side a north magnetic state. Whichever way, therefore, the copper tends to revolve on its own axis, the instant it moves, a power is evolved in such a direction as tends to stop its motion and bring it to rest. Being at rest in reference to this direction of motion, then there is no residual or other effect which tends to disturb it, and it remains still. 
2330. If the whole mass be moving parallel to itself, and be small in comparison with the face of the magnetic pole opposite to which it is placed, then, though it pass through the magnetic lines of force, and consequently have a tendency to the formation of magneto-electric currents within it, yet as all parts move with equal velocity and in the same direction through similar magnetic lines of force, the tendency to the formation of a current is the same in every part, and there is no actual production of current, and consequently nothing occurs which can in any way interfere with its freedom of motion. Hence the reason that though the rotation of the bar or cube (2324. 2328.) upon its own axis is stopped, its vibration as a pendulum is not affected.

2331. That neither the one nor the other motion is affected when the bar or cube is over the vertical pole (2328.), is simply because in both cases (with the given dimensions of the pole and the moving metal) the lines of particles through which the induced currents tend to move are parallel throughout the whole mass; and therefore, as there is no part by which the return of the current can be carried on, no current can be formed.

2332. Before proceeding to the explanation of the other phænomena, it will be necessary to point out the fact generally understood and acknowledged, I believe, that time is required for the development of magnetism in an iron core by a current of electricity; and also for its fall back again when the current is stopped. One effect of the gradual rise in power was referred to in the last series of these Researches (2170.). This time is probably longer with iron not well annealed than with very good and perfectly annealed iron. The last portions of magnetism which a given current can develope in a certain core of iron, are also apparently acquired more slowly than the first portions; and these portions (or the condition of iron to which they are due) also appear to be lost more slowly than the other portions of the power. If electric contact be made for an instant only, the magnetism developed by the current disappears as instantly on the breaking of the current, as it appeared on its formation; but if contact be continued for three or four seconds, breaking the contact is by no means accompanied by a disappearance of the magnetism with equal rapidity.

2333. In order to trace the peculiar effect of the copper, and its cause, let us consider the condition of the horizontal bar (2310. 2313.) when in the equatorial position, between the two magnetic poles, or before a single pole; the point of suspension being in a line with the axis of the pole and its ex- 
citing wire helix. On sending an electric current through the helix, both it and the magnet it produces will conduce to the formation of currents in the copper bar in the contrary direction. This is shown from my former researches (26.), and may be proved, by placing a small or large wire helix-shaped (if it be desired) in the form of the bar, and carrying away the currents produced in it, by wires to a galvanometer at a distance. Such currents being produced in the copper, only continue whilst the magnetism of the core is rising and then cease (18. 99.), but whilst they continue, they give a virtual magnetic polarity to that face of the copper bar which is opposite to a certain pole, the polarity being the same in kind as the pole it faces. Thus on the side of the bar facing the north pole of the magnet, a north polarity will be developed; and on that side facing the south pole, a south polarity will be generated.

2334. It is easy to see that if the copper during this time were opposite only one pole, or being between two poles, were nearer to one than the other, this effect would cause its repulsion. Still, it cannot account for the whole amount of the repulsion observed alike with copper as with bismuth (2295.), because the currents are of but momentary duration, and the repulsion due to them would cease with them. They do, however, cause a brief repulsive effort, to which is chiefly due the first part of the peculiar effect.

2335. For if the copper bar, instead of being parallel to the face of the magnetic pole, and therefore at right angles to the resultant of magnetic force, be inclined, forming, for instance, an angle of $45^{\circ}$ with the face, then the induced currents will move generally in a plane corresponding more or less to that angle, nearly as they do in the examining helix (2333.), if it be inclined in the same manner. This throws the polar axis of the bar of copper on one side, so that the north polarity is not directly opposed to the north pole of the inducing magnet, and hence the action both of this and the other magnetic pole upon the two polarities of the copper will be to send it further round, or to place it edgeways to the poles, or with its breadth parallel to the magnetic resultant passing through it (2323.) : the bar therefore receives an impulse, and the angle of it nearest to the magnet appears to be pulled up towards the magnet. This action of course stops the instant the mag* netism of the helix core ceases to rise, and then the motion due to this cause ceases, and the copper is simply subject to the action before described (2295.). At the same time that this twist or small portion of a turn round the point of suspension occurs, the centre of gravity of the whole mass is re- 
pelled, and thus $I$ believe all the actions up to this condition of things is accounted for.

2336. Then comes the revulsion which occurs upon the cessation of the electric current, and the falling of the magnetism in the core. According to the law of magneto-electric induction, the disappearance of the magnetic force will induce brief currents in the copper bar (28.), but in the contrary direction to those induced in the first instance; and therefore the virtual magnetic pole belonging to the copper for the moment, which is nearest the north end of the electromagnet, will be a south pole; and that which is furthest from the same pole of the magnet will be a north pole. Hence will arise an exertion of force on the bar tending to turn it round its centre of suspension in the contrary direction to that which occurred before, and hence the apparent revulsion; for the angle nearest the magnetic pole will recede from it, the broad face (2323.) or length (2315.) of the bar will come round and face towards the magnet, and an action the reverse in every respect of the first action will take place, except that whereas the motion was then only a few degrees, now it may extend to two or three revolutions.

2337. The cause of this difference is very obvious. In the first instance, the bar of copper was moving under influences powerfully tending to retard and stop it (2329.); in the second case these influences are gone, and the bar revolves freely with a force proportionate to the power exerted by the magnet upon the currents induced by its own action.

2338. Even when the copper is of such form as not to give the oblique resultant of magnetic action from the currents induced in it, when, for instance, it is a cube or a sphere, still the effect of the action described above is evident (2325.). When a plate of copper about three-fourths of an inch in thickness, and weighing two pounds, was sustained upon some loose blocks of wood and placed about 0.1 of an inch from the face of the magnetic pole, it was repelled and held off a certain distance upon the making and continuing of electric contact at the battery; and when the battery current was stopped, it returned towards the pole; but the return was much more powerful than that due to gravity alone (as was ascertained by an experiment), the plate being at that moment actually attracted, as well as tending by gravitation towards the magnet, so that it gave a strong tap against it.

2339. Such is, I believe, the explanation of the peculiar phænomena presented by copper in the magnetic field; and the reason why they appear with this metal and not with bismuth or heavy glass, is almost certainly to be found in its high 
electro-conducting power, which permits the formation of currents in it by inductive forces, that cannot produce the same in a corresponding degree in bismuth, and of course not at all in heavy glass.

2340. Any ordinary magnetism due to metals. by virtue of their inherent power, or the presence of small portions of the magnetic metals in them, must oppose the development of the results $I$ have been describing: and hence metals not of absolute purity cannot be compared with each other in this respect. I have, nevertheless, observed the same phænomena in other metals; and as far as regards the sluggishness of rotatory motion, traced it even into bismuth. The following are the metals which have presented the phænomena in a greater or smaller degree :-

Copper.
Silver.
Gold.
Zinc.
Cadmium.
Tin.

Mercury.
Platinum.
Palladium.
Lead.
Antimony.

2341. 'The accordance of these phænomena with the beautiful discovery of Arago*, with the results of the experiments of Herschel and Babbaget, and with my own former inquiries (81.) $\ddagger$, are very evident. Whether the effect obtained by Ampère, with his copper cylinder and a helix $\S$, was of this nature, I cannot judge, inasmuch as the circumstances of the experiment and the energy of the apparatus are not sufficiently stated; but it probably may have been.

2342. As, because of other duties, three or four weeks may elapse before I shall be able to complete the verification of certain experiments and conclusions, I submit at once these results to the attention of the Royal Society, and will shortly embody the account of the action of magnets on magnetic metals, their action on gases and vapours, and the general considerations in another series of these Researches.

Royal Institution, Nov. 27, 1845.

* Annales de Chimie, xxvii. 363; xxviii. 325; xxxii. 213. I am very glad to refer here to the Comptes Rendus of June 9, 1845, where it appears that it was $M$. Arago who first obtained his peculiar results by the use of electro- as well as common magnets.

$\dagger$ Philosophical Transactions, 1825, p. $467 . \quad \ddagger$ Ibid. 1832. p. 146.

$\oint$ Bibliothèque Universelle, xxi. p. 48. 